\title{
Different Traffic Patterns Over Ad Hoc Network Routing Protocols
}

\author{
Bindeshwar Singh Kushwaha \\ Banaras Hindu University \\ Department of Computer Science \\ Institute of Science \\ Varanasi, India
}

\author{
Pramod Kumar Mishra \\ Banaras Hindu University \\ Department of Computer Science \\ Institute of Science \\ Varanasi, India
}

\begin{abstract}
In this paper, we use three traffic generators namely exponential, Pareto and CBR (Constant Bit Rate) over an ad hoc network and analyze the behavior of routing protocols. Moreover, we take two parameters offered load and packet size which are important to determine the nature of ad hoc network routing protocols in the different traffic patterns. Further, simulation shows that there is an optimal value for which value of throughput and packet delivery ratio are optimal.
\end{abstract}

\section{General Terms}

Ad Hoc Network, Wireless Network

\section{Keywords}

Traffic Analysis, Pareto Traffic, Exponential Traffic, CBR traffic

\section{INTRODUCTION}

Recent years are seen as advance growth in wireless devices and extensive research in ad hoc network, and it seems that ad hoc network applications will be very useful in the near future. Then application layer traffic analysis becomes very important aspect of a protocol evaluation to make different types applications for ad hoc network. Moreover, actual communication takes place at application layer, and the different types of applications need different amount of resources. For instance, video streaming over the network requires more resources than sharing a file, sending an email needs accuracy but it does not require bandwidth or delay constraints. In addition, to meet these requirements the careful analysis of application traffic in the various conditions provides an insight to design routing protocol which can function according to applications requirement. For example, a cross layer path can be designed from the application layer to the network layer in which routing protocol will route packets according to traffic category or priority of the packet.

Furthermore, many researchers are working cross layer models to provide environment for video applications, in particular, real time traffic protocols RTP, VOIP will be the future's attractions.

\section{RELATED WORK}

Many researchers have analyzed impact of the transport layer and and network layer traffic on ad hoc network. In [2], UDP and TCP protocols are analyzed over the different traffic load. Furthermore, [5]|3] analyzed TCP traffic over ad hoc network. In [6, 7, 1] performance analysis of routing protocols is done. Additionally, in [4] there the mixed traffic of UDP and TCP is evaluated and a method is proposed to improve performance of TCP in presence of UDP. [8] observed the characteristics of CBR, FTP and Telnet traffic over ad hoc network routing protocols, [12] took a different approach to characterize behavior of the protocols, it set up multiple applications on the nodes which are in the network, and it also simulated peer to peer applications, as well as, in heavy data traffic. In addition, [13] carried out simulation at varying number of traffic sources using CBR traffic, another work [14] analyzed CBR over TCP in the different scenario. Equally important, [15] assessed MANET routing protocols under variable bit rate (VBR) and CBR traffics using different parameters. Further, [16] considered CBR source to conduct simulations over three MANET routing protocols. Moreover, in section [3] we explain different traffic generators, section [4] describes simulation environment and parameters we use to simulate network. Further, in section-[5][6] we analyze simulation results, and finally section-[7] concludes the paper.

\section{TRAFFIC GENERATORS}

In simulators, traffic generators [9] use probability distribution functions to generate application traffic [10] which have some patterns, and they have parameters, and value of parameters can be changed to evaluate a protocol.

\subsection{Exponential Probability Distribution Traffic}

Exponential distribution's PDF is defined as.

$$
f(x ; \lambda)= \begin{cases}\lambda e^{-\lambda x} & x \geq 0 \\ 0 & x<0 .\end{cases}
$$

Where $\lambda$ is the average exponential occurrence rate.

An on-off traffic can be generated by varying parameter $\lambda$. Packets can be emitted during on periods, and there is no packet emission 
during off period. In NS-2, burst time and idle time are taken from exponential distribution to generate application traffic.

The following parameters are need to be configured for traffic generation.

\begin{tabular}{|c|c|}
\hline Packet Size & Size of the packet to be generated \\
\hline Burst Time & On time for traffic generator \\
\hline Idle Time & Off time for traffic generator \\
\hline Rate & On time data rate \\
\hline
\end{tabular}

\subsection{Pareto Probability Distribution Traffic}

A random variable $\mathrm{X}$ is said to follow Pareto distribution, when it follows the following probability distribution function.

$$
F(x)=P(X>x)= \begin{cases}\left(\frac{x_{m}}{x}\right)^{\alpha} & x \geq x_{m} \\ 1 & x<x_{m}\end{cases}
$$

Where

$x_{m}$ is a scale parameter.

$\alpha$ is a shape parameter.

Using $x_{m}$ and $\alpha$ an on-off traffic can be generated by varying them.

The parameters [10] need to be configured to generate Pareto distribution traffic which are listed below.

\begin{tabular}{|c|c|}
\hline Packet Size & Size of the packet to be generated \\
\hline Burst Time & On time for traffic generator \\
\hline Idle Time & Off time for traffic generator \\
\hline Rate & On time data rate \\
\hline Shape & Shape parameter for distribution \\
\hline
\end{tabular}

\subsection{Constant Bit Rate Traffic}

Constant Bit Rate (CBR) traffic generator [10] emits packets at fixed bit rate, the traffic parameters which are to be configured, which are given.

\begin{tabular}{|c|c|}
\hline Packet Size & Size of the generated packet \\
\hline Data Rate & Rate at which data to be sent \\
\hline Interval & Time stamp between to packets \\
\hline
\end{tabular}

\section{SIMULATION ENVIRONMENT}

We consider [11] as simulator to asses network scenario. In addition, we place 40 nodes in 800 square meter, and pause time is 10 second, random waypoint is taken as mobility model. The maximum speed of nodes is 0.10 Meter/Second.

\begin{tabular}{|c|c|}
\hline Parameters & Value(s) \\
\hline Traffic Generators & CBR, Exponential, Pareto \\
\hline MAC Layer & $802.11 \mathrm{~b}$ \\
\hline Routing Protocol(s) & DSDV, DSR, AODV \\
\hline Transport Layer & UDP \\
\hline Mobility Model & Random Way Point \\
\hline Propagation Model & Two ray ground \\
\hline Simulation Area & 800 Meter Square \\
\hline Pause Time & 10 Second \\
\hline Number Of Nodes & 40 \\
\hline Maximum Speed & 0.10 Meter/Second \\
\hline
\end{tabular}

We take $802.11 \mathrm{~b}$ standard for the MAC and the physical layer, and UDP as transport layer protocol.

\section{THE IMPACT OF OFFERED LOAD ON THE DIFFERENT ROUTING PROTOCOLS}

\subsection{Throughput Analysis}

The network throughput is referred to as the average number of bits received by destination nodes in one second.

$$
\text { Throughput }=\frac{8 * T B}{T L B R-T F B S}
$$

\begin{tabular}{|c|c|}
\hline TB & Total Number of Bytes \\
\hline TFBS & Time at First Bit Sent \\
\hline TLBR & Time at Last Bit Received \\
\hline
\end{tabular}

It can be seen from the figure [1] when offered load is increased the application throughput of AODV and DSDV is also increased until the value offered load is $500 \mathrm{~KB} / \mathrm{sec}$, but after that the value of throughput starts staying constant. In this case, almost all the time the throughput of DSR routing protocol is constant.

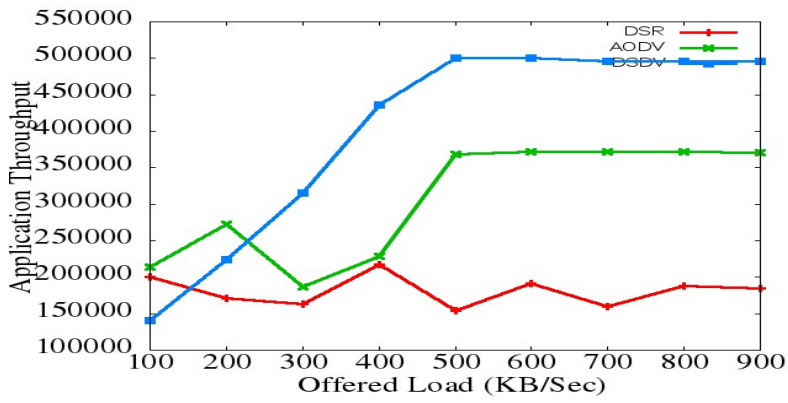

Fig 1: CBR Traffic

Under exponential traffic, from figure [2] the behavior of DSR is changed i.e. when offered load goes high the application throughput also goes high, but when it reaches at $500 \mathrm{~Kb} / \mathrm{sec}$ the value of the throughput started falling. In this case, AODV and DSDV also show the similar behavior.

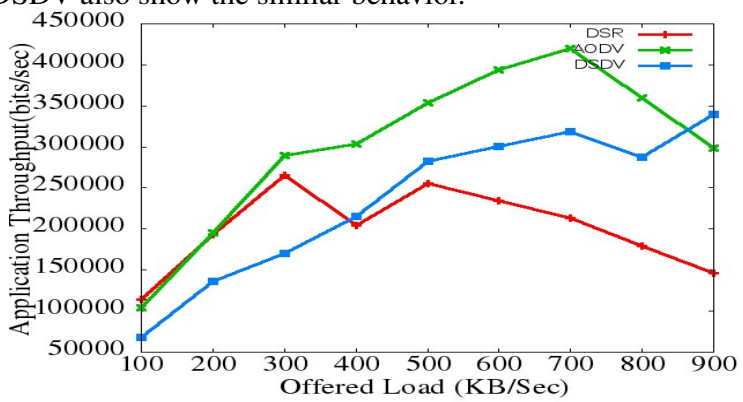

Fig 2: Exponential Traffic

In Pareto traffic, figure [3] AODV and DSR protocols have the same growing and falling pattern of throughput, but DSDV's throughput is substantially increased unless the value of offered load becomes $900 \mathrm{~Kb} / \mathrm{sec}$. 


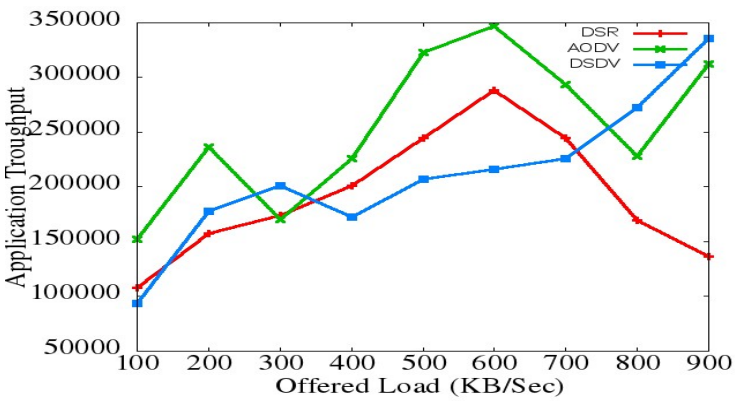

Fig 3: Pareto Traffic

After analyzing above three protocols, it can be concluded that when offered load is grew up throughput is also climbed, but after certain point it starts declining in all three traffic patterns. So, to achieve good throughput offered load should be balanced.

\subsection{Packet Delivery Ratio Analysis}

Packet Delivery Ratio

Packet delivery fraction is the ratio of packets received by destinations and sent by sources across the network.

$$
P D R=\frac{P r}{P s}
$$

\section{Where}

\begin{tabular}{|c|c|}
\hline Pr & Total Number of Packets Received \\
\hline Ps & Total Number of Packets Sent \\
\hline
\end{tabular}

Offered load also affects on packet delivery ratio. Figure [4] depicts that when offered load is low, packet delivery ratio is high. It may be because when load is low applications on the node generate small amount of packets, as a result, low overhead on the network, and chances of successful delivery of packets becomes high. But when offered is high, as a consequence, high overhead and, thus, grows in packet loss and low packet delivery ratio.

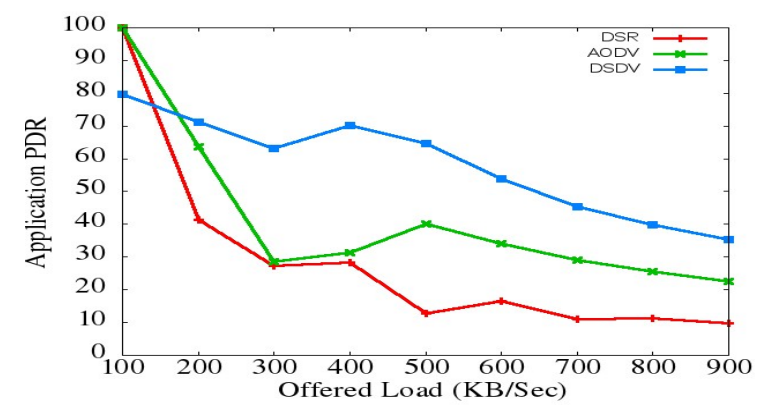

Fig 4: CBR Traffic

With reference to figure [5], DSR is more sensitive to offered load while DSDV is less affected compare to AODV and DSR.

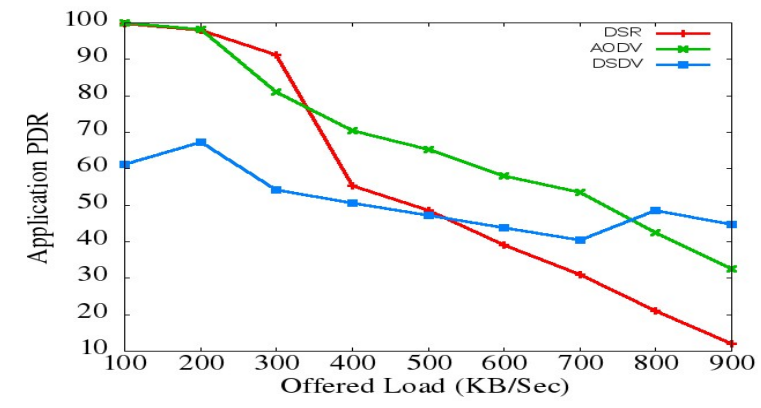

Fig 5:Exponential Traffic

Figure [6] shows almost the same pattern of AODV and DSDV the only difference that DSR is more sensitive in exponential than it was in CBR traffic.

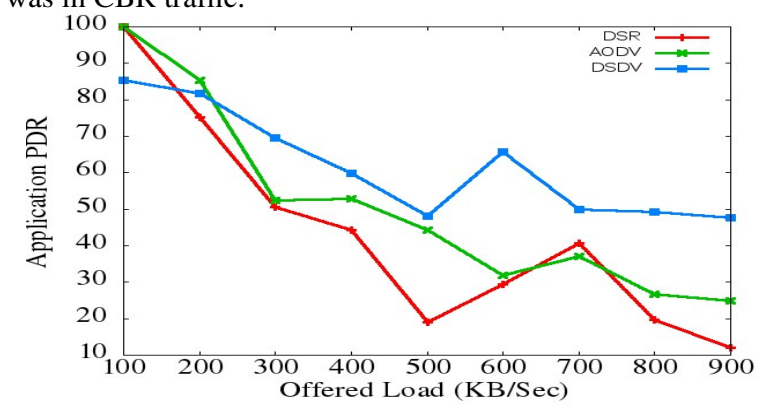

Fig 6:Pareto Traffic

\section{EFFECT OF PACKET SIZE ON THE DIFFERENT ROUTING PROTOCOLS}

\subsection{Throughput Analysis}

In CBR traffic, figure [7] the impact of the size of the packet on DSDV is null, there is no fluctuation at any point of the throughput's curve. Furthermore, AODV shows some inclines and declines DSR shows fluctuation at one point after that its throughput curve becomes steady.

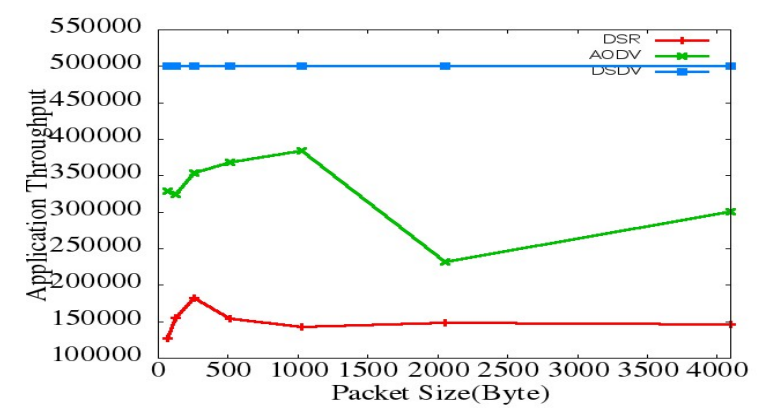

Fig 7:CBR Traffic

In case of exponential traffic, figure[8] when packet size is small the value of throughput is also small, but when size of packet is increased throughput of all the three protocols are grew up unless the value of packet size 512 bytes, but after the size of packet does not affect. So, packet size 512 bytes can be considered as optimized packet length in exponential traffic. 


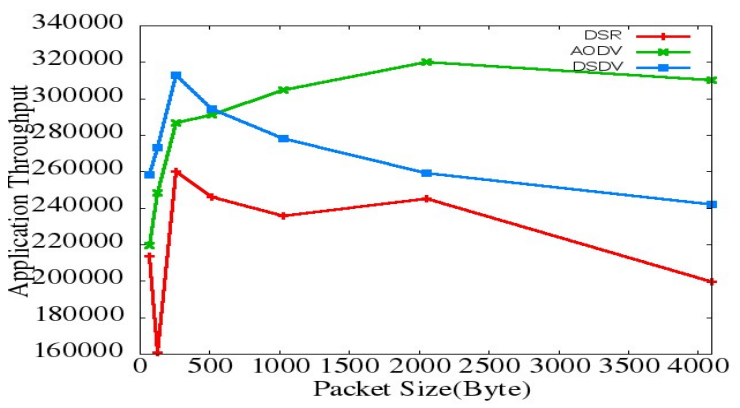

Fig 8: Exponential Traffic

In Pareto traffic pattern (figure [9]), there are fluctuations in all the three protocols unless packet size is 1024 bytes, then their throughputs are stayed.

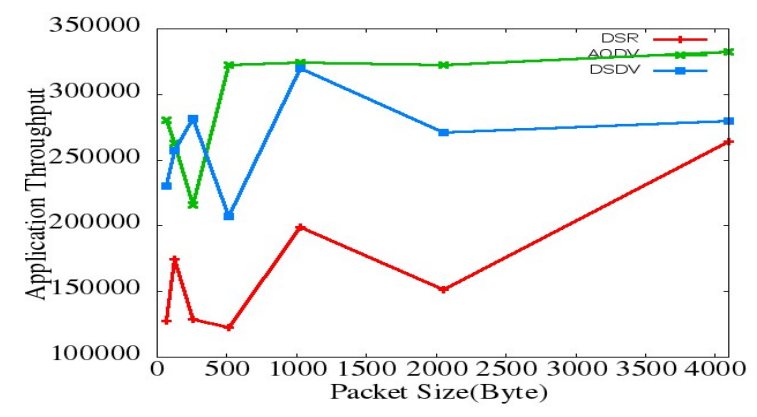

Fig 9: Pareto Traffic

After analyzing all three protocols, it can be said that there is a size of the packet for which throughput is optimal after that it becomes almost constant.

\subsection{PDR Analysis}

In CBR traffic when packet length is increased, there are also the increase in all three protocols, however, when size of packet is between 512 B to 1024 B either the grow in PDR is constant (in the case of DSDV) or there is a decrease (in the case AODV and DSR). So, there is also the optimal packet size for which PDR is optimum.

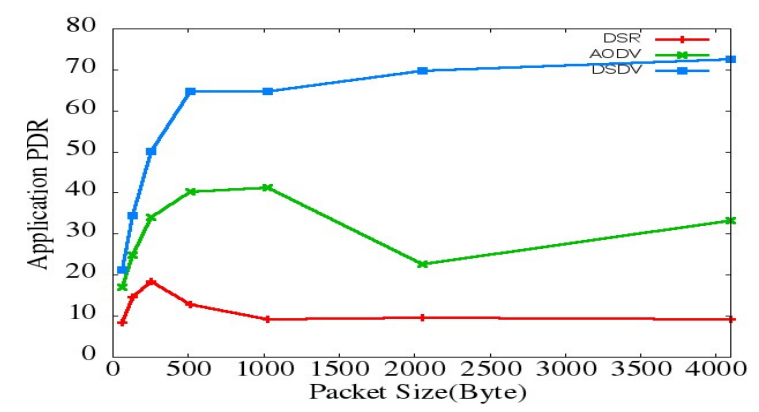

Fig 11: CBR Traffic

In case of exponential traffic, all protocol have the same performance except that there are no fall in the PDR value of AODV, DSDV.

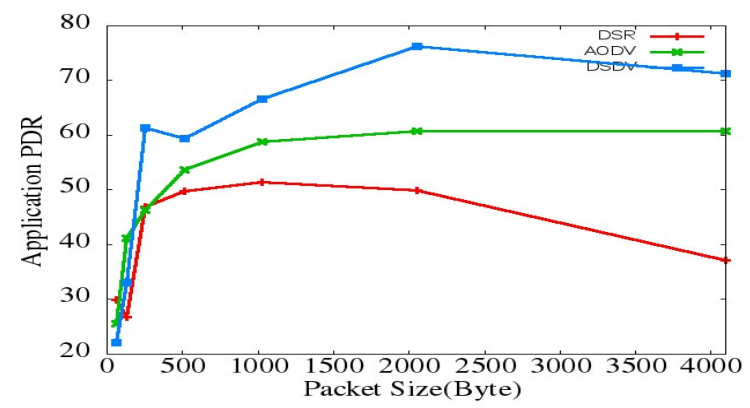

Fig 10: Exponential Traffic

There are fluctuations in PDR under the Pareto traffic, DSDV and DSR have the same fluctuation points, in the case of AODV its packet delivery ratio grows unless packet size is 512 Bytes then it becomes constant.

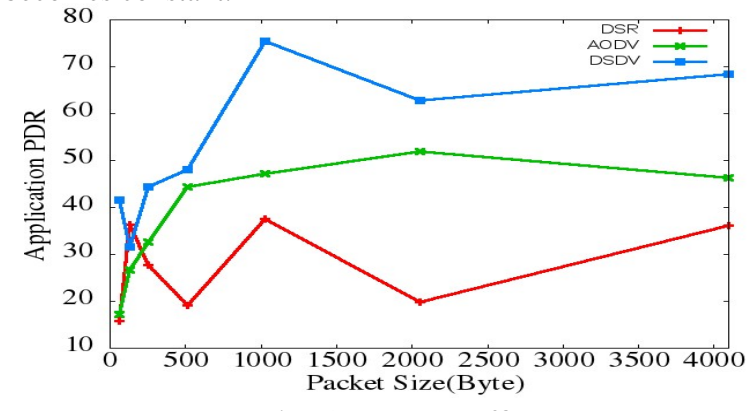

Fig 12: Pareto Traffic

\section{CONCLUSION AND FUTURE WORK}

To sum up, we can say that in all three protocols, DSDV is showing better performance than AODV and DSR, however, in exponential traffic AODV has better performance than DSDV. In addition, after analyzing all three protocols it can be observed that there are optimal values of packet size and offered load for which value of throughput and PDR values are optimal, after that their values are decreased or become constant. Moreover, cross layer design is one of the recent approaches to enhance the performance of the ad hoc network, and in future we have planned to design a cross layer information path from application layer to network layer to increase the performance of the network.

\section{REFERENCES}

[1] F. Bertocchi, P. Bergamo, G. Mazzini, M. Zorzi, Performance Comparison of Routing Protocols for Ad Hoc Networks GLOBECOM IEEE, 2003.

[2] T. Gopinath, A. S. Rathan Kumar, Rinki Sharma, Performance Evaluation of TCP and UDP over Wireless Ad Hoc Networks with Varying Traffic Loads, International Conference on Communication Systems and Network Technologies,2013.

[3] Thomas D. Dyer, Rajendra V. Boppana, A Comparison of TCP Performance over Three Routing Protocols for Mobile Ad Hoc Networks, ACM, 2001.

[4] Vikram Gupta, Srikanth V. Krishnamurthy, and Michalis Faloutsos, Improving the Performance of TCP in the Presence of Interacting UDP Flows in Ad Hoc Networks, LNCS 3042, pp. 64-75, 2004. 
[5] Gavin Holland, Nitin Vaidya, Analysis of TCP Performance over Mobile Ad Hoc Networks, Wireless Networks 8, 275?288, 2002.

[6] S. Mohapatra, P.Kanungo , Performance analysis of AODV, DSR, OLSR, and DSDV Routing Protocols Using NS2 simulator, International Conference on ation Technology Communication.

[7] J.Broch, D.Maltz, D.Johnson et al, A performance comparison of multi hop wireless ad hoc network routing protocols , 1998, 85-87.

[8] Vasudha Arora, C. Rama Krishna, Performance Evaluation of Routing Protocols for MANETs under Different Traffic Conditions, Second International Conference on Computer Engineering and Technology, 2010.

[9] Balakrishnan Chandrasekaran, Survey of Network Traffic Models http://www.cse.wustl.edu/ jain/cse56706/ftp/trafficmodels3/index.html.

[10] Application Objects for Traffic Generation, Tom Henderson http://www.isi.edu/nsnam/ns/.

[11] Network Simulator Version 2 Documentation, http://www.isi.edu/nsnam/ns/.

[12] Himabindu Pucha Saumitra M. Das Y. Charlie Hu, The Performance Impact of Traffic Patterns on Routing Protocols in Mobile Ad Hoc Networks, MSWiM?04 October 4-6, 2004.

[13] Sabina Barakovi, Jasmina Barakovi, Comparative Performance Evaluation of Mobile Ad Hoc Routing Protocols, MIPRO 2010.

[14] Rachit Jain, Naresh B. Khairnar, Laxmi Shrivastava, Comparative Study of Three Mobile Ad-hoc Network Routing Protocols under Different Traffic Source, ICCSNT 2011.

[15] Qutaiba Razouqi, Ahmed Boushehri, Mohamed Gaballah, Lina Alsaleh, Combined Traffic Simulation Scenarios Performance Investigation Routing protocols AODV, DSR and DSDV in MANET, IEEE Conference, 2012.

[16] Yudhvir Singh, Amit Kuma, Prabha Rani, Sunil Kumar Kaushik, Impact of CBR Traffic on Routing Protocols in MANETs, UKSim-AMSS 16th International Conference on Computer Modelling and Simulation 2014. 